\title{
Energy-aware strategy for data forwarding in IoT ecosystem
}

\author{
K. Nagarathna
}

Electronics and Communication Department, Basaveshvara Engineering College, India

\begin{tabular}{l} 
Article Info \\
\hline Article history: \\
Received Oct 3, 2019 \\
Revised Mar 12, 2020 \\
Accepted Mar 30, 2020 \\
\hline Keywords: \\
Energy consumption \\
Energy-efficiency \\
IoT \\
Routing protocol \\
SDN controller
\end{tabular}

\begin{abstract}
The Internet of Things (IoT) is looming technology rapidly attracting many industries and drawing research attention. Although the scale of IoT-applications is very large, the capabilities of the IoT-devices are limited, especially in terms of energy. However, various research works have been done to alleviate these shortcomings, but the schemes introduced in the literature are complex and difficult to implement in practical scenarios. Therefore, considering the energy consumption of heterogeneous nodes in IoT eco-system, a simple energy-efficient routing technique is proposed. The proposed system has also employed an SDN controller that acts as a centralized manager to control and monitor network services, there by restricting the access of selfish nodes to the network. The proposed system constructs an analytical algorithm that provides reliable data transmission operations and controls energy consumption using a strategic mechanism where the path selection process is performed based on the remaining energy of adjacent nodes located in the direction of the destination node. The proposed energy-efficient data forwarding mechanism is compared with the existing AODV routing technique. The simulation result demonstrates that the protocol is superior to AODV in terms of packet delivery rate, throughput, and end-to-end delay.
\end{abstract}

Copyright $\odot 2020$ Institute of Advanced Engineering and Science. All rights reserved.

\section{Corresponding Author:}

K. Nagarathna,

Electronics and Communication Department,

Basaveshvara Engineering College,

Bagalkot, India.

Email: nagarathnavtu@gmail.com

\section{INTRODUCTION}

The internet of things-(IoT) is a group of structures that enable new forms of networking to provide cost-effective, fast data sharing and communication services in various application areas. It represents a network containing heterogeneous devices-(Things) to serve intelligent services to users. A lot of research and development work has been done in the field of Internet of Things [1]. With this development, the IoT is attracting massive attention from many application areas like smart home, smart security system, smart road traffic monitoring, healthcare systems, and many more [2,3]. The IoT network is massive and complex and contains things like RFID tags, smartphones, and wireless sensors for capturing data from the surroundings. Since IoT consists of heterogeneous networks and most devices in IoT typically have lower computing capacity, lower processing power, and limited battery power [4].

Power consumption is one of the critical concerns for the seamless data transmission process. Information relay from the source point to the target point is one of the vital operations to be performed in a large-scale dynamic IoT environment. Due to the limited transmission range of sensors, the sensor forwards the collected data through the intermediate node. An intermediate node then utilizes unintentional power for the data transmission from the source node, which leads to higher energy depletion and thereby accelerates factor to cause network lifetime shorter. Therefore, efficient power utilization by nodes is a significant factor affecting performances of the network in the IoT ecosystem [5]. Hence, an optimal approach and efficient 
routing strategies are required to assists long-term inter-communication among the different devices. Also, the data or packet transmission should be executed in such a way that it reaches its destination using the most suitable or shortest path.Various research worksand efforts are made in past few years to address energy problem in smart sensor networks [6-8].

Existing wireless sensor routing protocols are inherently complex, requiring extensive use of processing power and memory, which is naturally a limited resource in devices involved in IoT network. If a large number of route discoveries are initiated, the energy of nodes in the network quickly drains out because it requires an unnecessarily forwarding of a large number of route-requests. It has also been seen that most of the existing routing techniques use hop count to determine the shortest route between source and destination nodes. For dynamic topologies in the IoT, it is not feasible to use hop counts only as routing metrics because it does not consider many sensitive factors such as packet loss, link quality, data rate, channel diversity, etc $[9,10]$. Therefore, there is a need for a cost-effective and straightforward routing mechanism that can effectively save IoT devices energy.

This paper introduces a simple and energy-efficient routing protocol with an SDN controller in the network that acts as a unified leader providing security and power-aware services by rejecting access to selfish nodes in the network. Here, the SDN controller knows the topology in the entire network and identifies the best route to transfer data from source to destination node. The proposed paper is organized as follows: Section 1 presents related works towards optimizing energy consumption also describes the problem statement for the proposed study. Section 2 presents the design of the proposed system. Section 2 presents the algorithm description. Results and discussions are presented Section 3. Finally, the conclusion is presented in Section 4.

The Background. Several studies have been done for addressing the routing issues in IoT based applications. The work done by Tseng [11] has designed a multipath load normalizing routing mechanism to address bottleneck problem caused by heavy data transmission, in sensor networks. However, this work has not taken into account the load balancing factor for the bursty traffic. Al-Janabi et al. [12] have introduced Software Define Network-(SDN)-supported scalable and optimal routing protocol-oriented BS path supervision mechanism for a large IoT ecosystem. In this, the authors used a combined approach of genetic algorithms (GA) and Particle Swarm Optimization-(PSO) techniques to establish suitable paths for data forwarding and node communication. In the study of Oh and Lim [13], a cost-effective routing protocol was designed using Bloom filters and rank principles to boost the robustness and energy efficiency in the network.

The study done by Bouaziz et al. [14] considers the challenges posed by device mobility and energy-aware routing protocol is designed to minimize the negative impact caused by intermittent node connections and link interruption factor. This approach also incorporates a mobility recognition and replacement policy that is aware of resource constraints to enhance network performance concerning energy efficiency and link reliability. S.Vasiliev et al. [15] have studied about the throughput capabilities of relaying methods with ARQ system in Flying Ad hoc Networks-(FANET). The authors here present Markov models of multiple relaying algorithms and performed a comparative assessment of the throughput efficiency of such relying techniques. In the study of Xiang et al. [16], a power-aware routing algorithm for SDN-WSN is investigated by choosing control nodes. These control nodes dynamically assign different jobs to others based on their residual energy. However, it is challenging to identify the precise number of nodes required and the number of nodes under the direction of a control node.

The work done by Gante et al. [17] towards network management, introduced a smart management scheme by adopting the OpenFlow protocol and modified forwarding rule to perform optimal communication between all nodes throughout the network. Luo et al. [18] designed an SDN oriented WSN architecture to address issues of policy inflexibility and its management by improving Sensor OpenFlow. Jacobsson and Orfanidis [19] used low-cost hardware design and an efficient placement technique to reconfigure WSN architecture and its functionality. The work of Haseeb et al. [20] constructed a power-efficient and robust multi-hop routing mechanism based on secret-key allocation strategy to boost the performance of network against malicious behavior. The study outcome demonstrates the effectiveness of the presented routing mechanism from the perspective of network life-span and throughput.

The work of Khan et al. [21], considers issues related to energy efficiency enhancement in underwater sensor networks. In order to solve such problems, the authors provide a dual-tire cluster oriented efficient routing protocol that is mainly designed to deal with a high error rate, high consumption of energy, and end-to-end delay. The study outcome suggests that the presented approach achieves superior performance compared to other similar existing approaches regarding network life-span, reliable data transmission. Similarly, the work of Nguyen et al. [22] studied the energy and QoS issues in heterogeneous IoT networks and developed an energy harvesting oriented routing technique to solve the issues associated with unpredictable traffic load changes and the remaining energy conditions. The outcome exhibits that 
the introduced technique can effectively provide a practical mechanism to improve power efficiency while meeting QoS requirements. In the study of Kumar and Vidyarthi [23], a green routing mechanism is designed based on Fork and PSO to enhance the longevity of the sensor network. This algorithm optimizes control nodes and its clustering. The study outcome illustrates that the presented mechanism is better than other existing approaches in terms of prolonged network service life-span.

Din et al. [24] presented an enhanced mechanism for route selection to cluster head-( $\mathrm{CH})$ oriented on multi-hop routing in multi-layer WSN. This mechanism identifies the route on the basis of minimum cost between multiple connected routes. The study outcomes demonstrate the effectiveness of the presented approach regarding prolong network service duration. Lin et al. [25] presented a fault-tolerant routing mechanism for IoT WSN. The author has used the concept of virtual CH establishment and flow-graph architecture for bearing $\mathrm{CH}$ failure effectively. All resources for the non-faulty $\mathrm{CH}$ are ordered as virtual $\mathrm{CHs}$ as a backup for all failed CHs. Then, after a concept of flow-graph architecture is modeled to obtain fault tolerance property while reducing overall power consumption in all non-faulty CHs. The study outcome exhibits the potential of the presented fault-tolerant routing mechanism regarding network life-span enhancement.

An efficient routing protocol has been introduced in the study of Selem et al. [26] for the wireless body area network (WBAN) in IoT supported health application. This approach is designed to address the problem related to the network performance and to control the damaging effects on human skin caused due to elevated body sensors temperature. The experimental outcome exhibits that the presented technique maintains better network performances regarding node life and packet throughput. The study of Naggarathna and Mallapur [27-31] have addressed time synchronization and routing problem and presented delay-aware routing scheme to design optimal strategy that compensates delay factor in large scale WSN. Based on result analyis the presented study is found to be effective for reducing overhead, synchronization errors, and processing time when compared with the existing time synchronization methods.

Begam and Prema [32] designed energy-harvesting-based routing technique. In this, a concept of the artificial neural network is adopted for the training. The experimental result of this study demonstrates that an introduced technique improves node life-span, QoS, and entire network service duration under the influence of dynamic factors such as uncertain traffic load and residual energy conditions. Tian et al. [33] introduced the energy-aware strategy for industrial IoT applications, which combines network coding technique and power control schemes to minimize energy consumption by reducing burden in the packet transmission process and at the same time allowing sensor nodes to select suitable transmit energy. The work carried out by Sobral et al. [34] suggested a solution to improve the performance of existing LOADng routing protocol in mobile IoT. The study presented a mechanism that allows sensor nodes to understand the availability of their adjacent nodes using control messages so that these sensor nodes can choose a short route and avoid forwarding data through interrupted paths due to node mobility.

The Problem Description. IoT applications have predicted the future where end-users, wireless communication technologies, and computing systems, will provide a variety of capabilities to achieve high economic efficiency and convenience. However, such diverse applications face various difficulties due to constraint nature of devices in IoT-ecosystem. The major challenge associated with IoT is the optimal energy utilization in the communication process that involves heterogeneous devices connected via the Internet. Also, these devices are typically distributed in areas with different levels of heterogeneity. The energy used to transmit data depends on several attributes, such as the distance from the node to the target point, the size of the data packet to be transmitted, the energy amplification cost before transmission and the energy consumed in the process of event sensing, data capturing and node communications. However, various routing protocols have been introduced so far, for enhancing network performance and reducing energy consumption. But there is not a single protocol that is accepted global standard for IoT.

The solutions presented in the literature are complex and difficult to implement in real-time situations. Also, some of the existing techniques towards energy minimization are designed considering particular issues they do have not considered other factors like energy hole issue in the network which is also a critical factor that can affect overall network performance. Therefore, preserving node power is a critical requirement considering multiple factors for the longer network lifetime. The problem statement for this study can be expressed as "designing a very simple and optimal technique is a challenging task that can enable energy-efficient data transfer and reliable communication between heterogeneous devices and maintains long network service time".

The proposed system. The key objective of the proposed study is to present a new power savings mechanism while exhibiting routing and packet transmission operations in IoT applications. Therefore, the study introduces an SDN assisted efficient routing strategy that allows IoT nodes with different and limited energy to be interconnected. The adoption of SDN concept offers a secure and energy-aware network 
by denying insensitive to be participating in the communication operation of the network. A schematicdesign of the proposed system is shown in Figure 1.

The proposed study has adopted an analytical methodology to implement SDN-based control systems that can perform secure and energy-efficient routing. The development of the proposed system is carried out with some considerations that in IoT ecosystem nodes are heterogeneous with different energy values, and each node is synchronized with the SDN control system. The SDN controller system is also responsible for executing other varieties and essential services that make network performance better and flexible. Therefore, IoT nodes in the network directly do not perform any communication process, but via the SDN controller, the nodes can establish a connection among themselves based on the forwarding decisions. For this, every active node in the network is assigned with a unique identity.

An Open Flow protocol is adopted that set up the communication between an SDN controller and the nodes in the network. The controller has the right to perform multiple administrative jobs like it can perform elimination and update of traffic flow according to forwarding rules. Apart from this, it performs some additional network services like topology discovery, routing, traffic control, policy management, and access control and energy management. However, it is possible that some nodes lacks a sufficient amount of energy and become very weak or insensitive. The SDN controller can easily recognize the insensitive nodes because it has all details about the network and constantly monitors the nodes. If the node is found to be below a certain cut-off or threshold value, the node is then considered as an insensitive node and is blacklisted in the table maintained by the SDN controller. Once the node is blacklisted, then it will not be able to participate in the communication process and will not access the network services. If any node tries to access the network, the controller checks its Id in the blacklist.

For packet forwarding operation, the source node first discovers information about neighbour nodes. The source node will broadcast path request message PREQ in every direction to determine its adjacent nodes. All other nodes located near the source node will receive PREQ and sends a path reply message PREP to forward the packet from source to the traget node. However, it is quite likely that the source node may receive PREP message from multiple adjacent nodes that may or may not located in the direction of the destination node. This can lead to the generation of control message overhead problem. Therefore, the study adopts the timeout mechanism through SDN control to avoid the overhead issue and to achieve a single response with appropriate information about its adjacent nodes located in the direction of the destination node. An energy analyser module is also implemented by SDN controller to determine the residual energy status of each adjacent node if the energy of the node is below the cut-off-value, the packet is sent, and otherwise, another neighbouring node is selected [35-39]. Table 1 is maintained by a controller that contains all information about the nodes for the packet transmission.

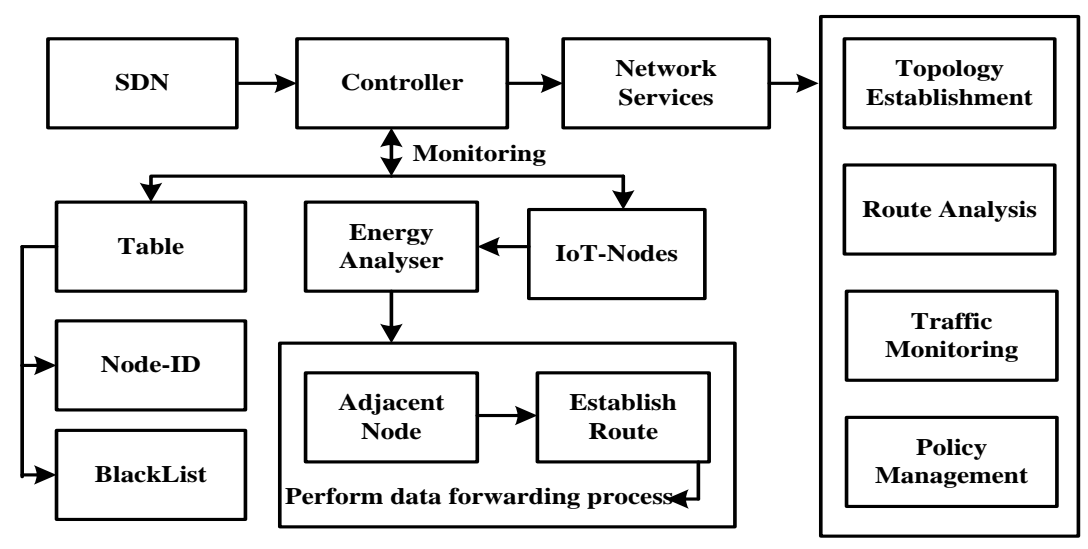

Figure 1. Proposed system architecture

Table 1. Information maintained by SDN controller

\begin{tabular}{ccc}
\hline Sn. No & Details & Notations \\
\hline 1 & IoT-Nodes-Id & N-Id \\
2 & Source Node-Id & Ni-Id \\
3 & Destination Node-Id & N $_{\text {D-Id }}$ \\
4 & Residual energy & R-Id \\
5 & Threshold & TH \\
6 & BlackList Node-Id & BL-N \\
7 & Adjacent Node-Id & Adj-Id \\
\hline
\end{tabular}




\section{ALGORITHM DESCRIPTION}

This section presents an algorithm description for the implementation of the proposed methodology for achieving energy-aware packet transmission operation. The significant steps in implementation are mentioned as follows:

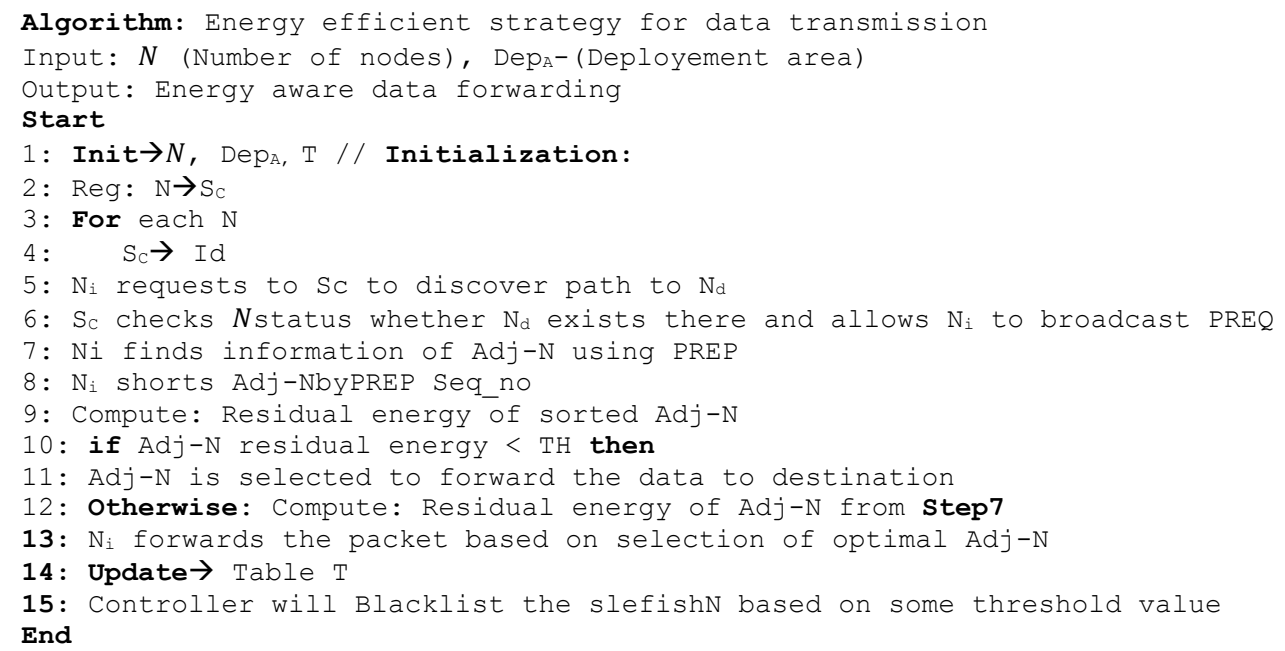

The above-demonstrated computing steps are responsible for establishing an energy-efficient strategy for forwarding data packets from source node-(Ni) to destination node-(Nd). The first step of the algorithm is subjected to initialization of nodes-(N) and deployment area-(DepA) (Step-1). In the next step, all nodes in the deployment area are registered to SDN controller-(SC) (Step2). The SC then assigns a unique id for each node in the network which is maintained by the controller in the table T (Step-3 and 4). For the data forwarding process, the $\mathrm{Ni}$ makes a request to $\mathrm{SC}$ for initiating path discovery process to destination node-(Nd)-(Step-5). The SC checks the status of the network and ensures whether destination node $\mathrm{Nd}$ exists there and allows $\mathrm{Ni}$ to broadcast path request message PREQ to find all possible number of adjacent nodes-(Adj-N) that can participate in packet transmission operation (Step-6).

The Ni receives path reply message PREP and finds information about their adjacent nodes-(Adj-N) (Step-7). Also, it is possible that the Ni may receive irrelevant PREP message from the multiple Adj-N that may not be located in the direction of Nd. Therefore, the timeout mechanism is implemented by SC to avoid overhead issue caused by irrelevant PREP messages. In step 8, a simple sorting mechanism is applied to short the PREP based on their sequence number to obtain the most suitable number of Adj-N. This process also preserves energy by avoiding the multiple Adj-Ns that are unnecessary participating in the data transmission process. After residual energy of selected Adj-Ns between $\mathrm{Ni}$ and $\mathrm{Nd}$ is computed-(Step-9) and is evaluated with a threshold value to forward data (Step10).

If the remaining energy evaluation condition is met, $\mathrm{Adj}-\mathrm{N}$ is selected to forward the data to the destination (step 11), otherwise the system proceeds to step 7, and computes residual energy of all computed Adj-Nsand based on their residual energy level it will be finally selected to participate in the packet transmission process from Ni to Nd-(Step-12 and 13). All the nodes that are selected to participate in packet transmission process and their residual energy value is stored in the table $\mathrm{T}$. Apart from this the controller also saves id of selfish node (i.e., that have low energy and are vulnerable to security attacks) into the blacklist based on some cut-off value and also it can not be able to access the network operation further. This process helps in maintaining seamless services across the network for packet transmission and node communication without any disruption. The flow diagram of the entire methodology for obtaining energy-aware packet transmission strategy in IoT operation is demonstrated as Figure 2.

Energy is consumed in both the transmission and reception of the data. The following variables are used to derive the energy equation for the energy model:

Transmitting energy- $\left(\mathrm{T}_{\mathrm{E}}\right)$

$$
T_{E}=\text { Data }_{P c k t} \text { Size } * E_{T R}+E_{\alpha d} * d
$$

where,

$d=d^{2}$ for short distanceq

$d=d^{4}$ for long distancew 
Receiving Energy- $\left(\mathrm{R}_{\mathrm{E}}\right)$

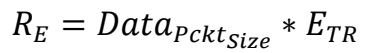

Current level of Eenergy-( $\left.\mathrm{E}_{\text {curnt }}\right)$

$$
E_{\text {Curnt }}=T_{E}+R_{E}
$$

Residual Energy-( $\left.E_{\text {res }}\right)$

$$
E_{\text {res }}=E_{\text {initial }}-E_{\text {Curnt }}
$$

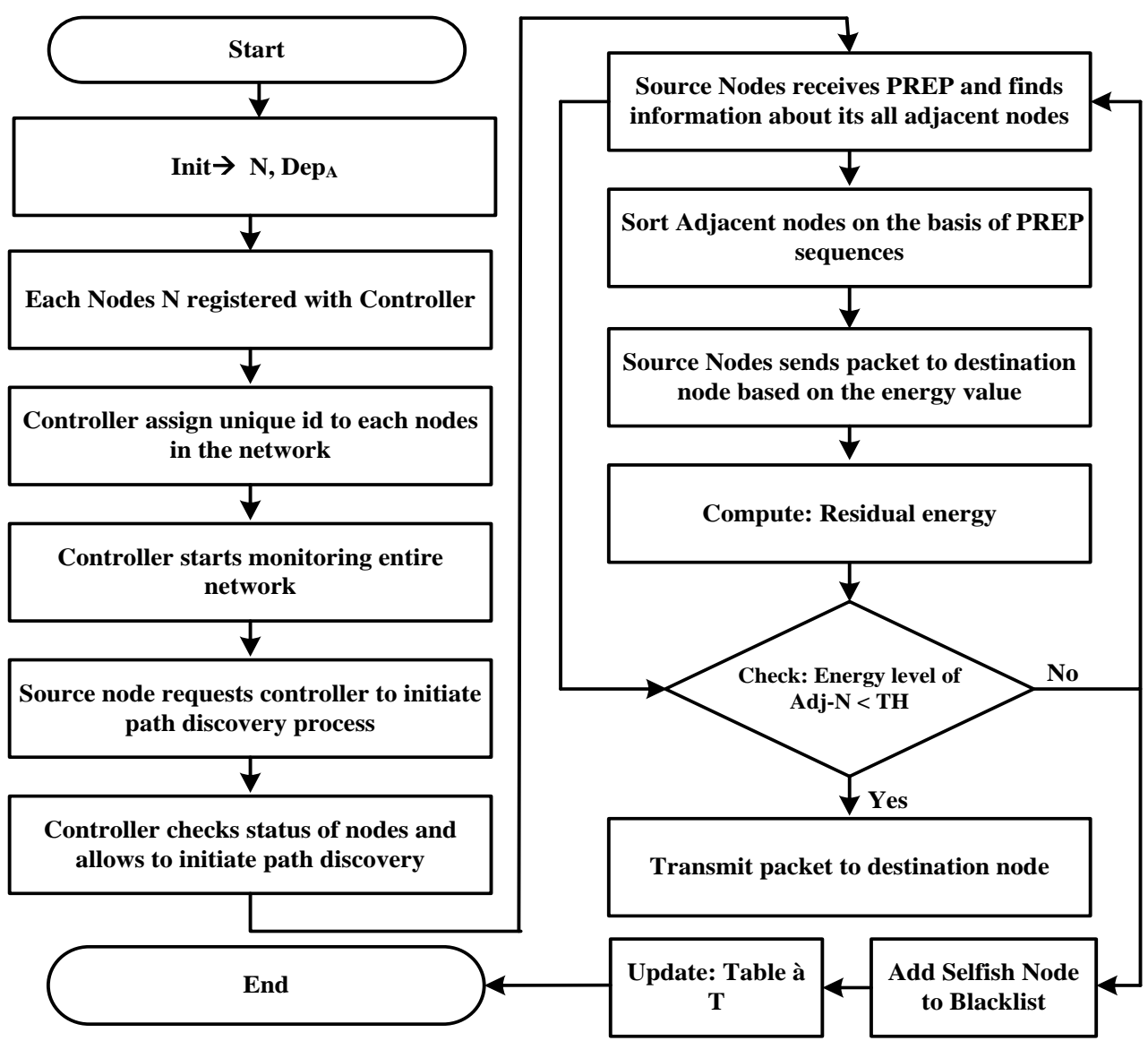

Figure 2. Flow diagram of energy aware packet transmission operation

\section{RESULTS AND ANALYSIS}

This section presents the outcome analysis of the proposed methodology for illustrating the effectiveness of the proposed system. The implementation of proposed energy-efficient data forwarding methodology is carried on the numerical computing platform. The performance evaluation of the proposed energy-efficient method is evaluated in terms of packet delivery rate-(PDR), Throughput, and Mean end-to-end delay-(MEED). The superiority of proposed methods is analysed based on the comparative analysis with existing AODV protocol. The simulation parameters that are used for performing the experimental task is demonstrated in Table 2.

The comparative analysis demonstrates the effectiveness of the proposed system. The performance of the proposed system in term of PDR is shown in Figure 3. PDR is found to be better when compared to the existing AODV protocol. In initial steps, the existing AODV shows better performance but further with an increase of nodes the proposed system performs better than the AODV. The throughput comparison is demonstrated in the Figure 4. Throughput is also found to be improved when compared to the existing 
techniques. The end-to-end delay performance analysis demonstrated in Figure 5. In first 20s AODV shows slightly better performance than the proposed system, but the overall delay is found be decreased compared to the existing techniques system.

Table 2. Simulation parameters

\begin{tabular}{ccc}
\hline Sn. No & Parameters & Values \\
\hline 1 & Tool & MatLab \\
2 & System & Windows 10 \\
3 & Number of Nodes & 70 \\
4 & Simulation round & 300 \\
5 & Deployment area & $500 \mathrm{~m} \mathrm{x} 500 \mathrm{~m}$ \\
6 & Communication range & $50 \mathrm{~m}$ \\
7 & MAC Protocol & 802.11 \\
8 & Energy Model & Radio \\
9 & Packet size & 1000 bytes \\
10 & E $_{\text {init }}$ & $10 \mathrm{~J}$ \\
11 & Ini $_{\text {Trust }}$ & 5 \\
\hline
\end{tabular}

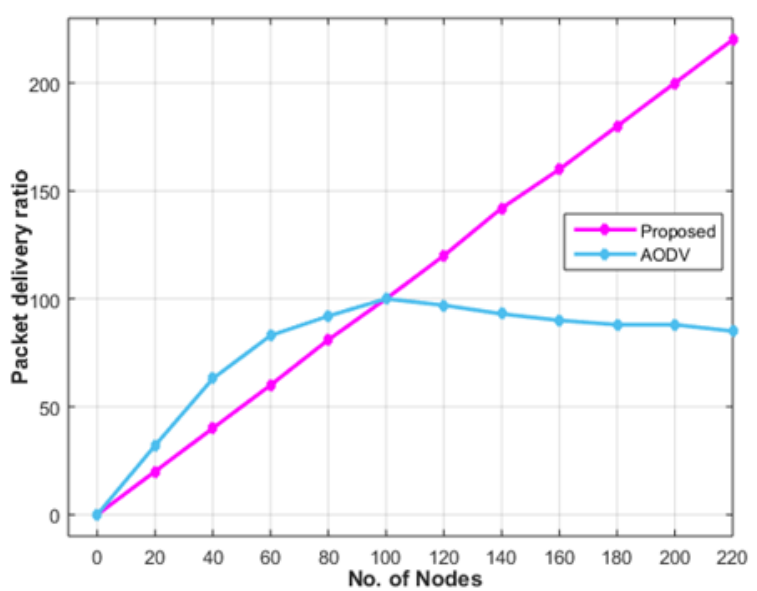

Figure 3. Packet delivery ratio analysis

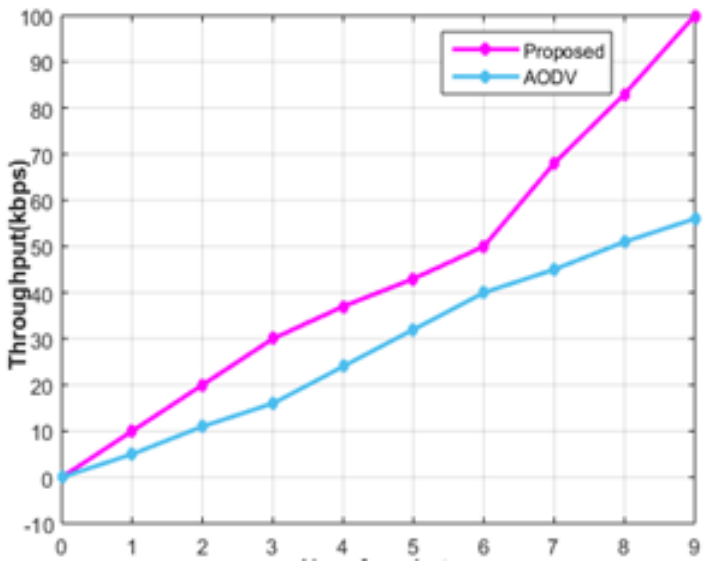

Figure 4. Throughput analysis

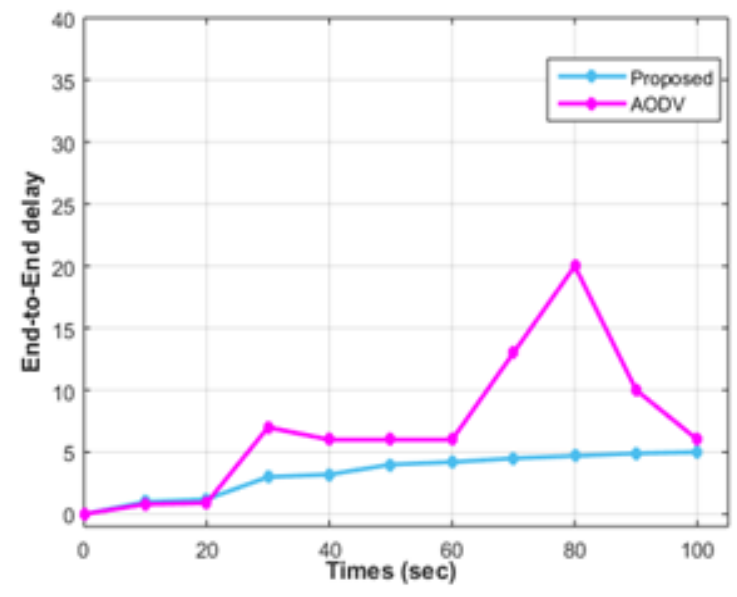

Figure 5. End-to-end delay analysis

\section{CONCLUSION}

In this paper, an energy-efficient routing strategy is proposed for IoT eco-system, which adopts SDN Controller with optimal route selection mechanism for the data transmission. The proposed analytical algorithm provides reliable data transmission operations and controls the energy consumption by using 
an optimal mechanism for routing selection and analysing the remaining energy of the adjacent node for the packet transmission. The results exhibit that the proposed energy-aware data forwarding strategy outperforms the existing AODV routing technique and improves the overall performance of the network in terms of packet-delivery rate, end-to-end delay, and throughput.

\section{REFERENCES}

[1] P. V. Dudhe, et al., "Internet of Things (IOT): An overview and its applications," 2017 International Conference on Energy, Communication, Data Analytics and Soft Computing (ICECDS), pp. 2650-2653, 2017.

[2] P. Sethi and S. R. Sarangi, "Internet of things: architectures, protocols, and applications," Journal of Electrical and Computer Engineering, vol. 2017, no. 1, pp. 1-25, 2017.

[3] A. Bhushan and P. Garg, "Internet of Things: A Review on its Future Scope \& Challenges," National Conference on Recent Advancement in Engineering \& Science, 2015.

[4] D. H. Mun, et al., "An assessment of internet of things protocols for resource-constrained applications," IEEE 40th Annual Computer Software and Applications Conference (COMPSAC), vol. 1, pp. 555-560, 2016.

[5] F. Jammes, "Internet of Things in Energy Efficiency: The Internet of Things (Ubiquity symposium)," Ubiquity, vol. 2016, no. 2, Feb 2016.

[6] I. Ud Din, et al., "The Internet of Things: A review of enabled technologies and future challenges," IEEE Access, vol. 7, pp. 7606-7640, 2019.

[7] J. Cabra, et al., "An IoT approach for wireless sensor networks applied to e-health environmental monitoring," 2017 IEEE International Conference on Internet of Things (iThings) and IEEE Green Computing and Communications (GreenCom) and IEEE Cyber, Physical and Social Computing (CPSCom) and IEEE Smart Data (SmartData), pp. 578-583, 2017.

[8] O. Elijah, et al., "An overview of Internet of Things (IoT) and data analytics in agriculture: Benefits and challenges," IEEE Internet of Things Journal, vol. 5, no. 5, pp. 3758-3773, 2018.

[9] M. Patil and S. Gundagatti, "A Brief Survey on QoS based Routing Protocols for Real Time Multimedia Transmission in Wireless Systems," Advances in Wireless and Mobile Communications, vol. 11, no. 1, pp. 31-41, 2018.

[10] H. P. Alahari and S. B. Yalavarthi, "A survey on network routing protocols in internet of things (IOT)," International Journal of Computer Applications, vol. 160, no. 2, pp. 18-22, 2017.

[11] C. H. Tseng, "Multipath load balancing routing for Internet of Things," Journal of Sensors, vol. 2016, pp. 1-8, 2016.

[12] T. A. Al-Janabi and H. S. Al-Raweshidy, "A centralized routing protocol with a scheduled mobile sink-based AI for large scale I-IoT,” IEEE Sensors Journal, vol. 18, no. 24, pp. 10248-10261, 2018.

[13] H. Oh and S. Lim, "Light-weight Routing Protocol in IoT-based Inter-Device Telecommunication Wireless Environment," International Journal of Electrical and Computer Engineering, vol. 6, no. 5, pp. 2352-2361, 2016.

[14] M. Bouaziz, et al., "EC-MRPL: An energy efficient and mobility support routing protocol for Internet of Mobile Things," in The 14th Annual IEEE Consumer Communications and Networking Conference, pp. 19-24, 2017.

[15] D. S. Vasiliev and A. Abilov, "Relaying algorithms with ARQ in flying Ad hoc networks," in 2015 International Siberian Conference on Control and Communications, SIBCON, pp.1-5, 2015.

[16] W. Xiang, et al., "An energy-efficient routing algorithm for software-defined wireless sensor networks," IEEE Sensors Journal, vol. 16, no. 20, pp. 7393-7400, 2016.

[17] A. DeGante, et al., "Smart wireless sensor network management based on software-defined networking," in Proceedings of the 2014 the 27th Biennial Symposium Communications, Kingston, ON, Canada, pp. 71-75, 2014.

[18] T. Luo, et al., "Sensor open flow: Enabling software-defined wireless sensor networks," IEEE Communication Letters, vol. 16, no. 11, pp. 1896-1899, 2012.

[19] M. Jacobsson and C. Orfanidis, "Using software-defined networking principles for wireless sensor networks," in Proceedings of the 11 $11^{\text {th }}$ Swedish National Computer Networking Workshop (SNCNW), Karlstad, Sweden, 2015.

[20] K. Haseeb, et al., "Secret Sharing-Based Energy-Aware and Multi-Hop Routing Protocol for IoT Based WSNs," IEEE Access, vol. 7, pp. 79980-79988, 2019.

[21] W. Khan, et al., "A Multi-Layer Cluster Based Energy Efficient Routing Scheme for UWSNs," IEEE Access, vol. 7, pp. 77398-77410, 2019.

[22] T. D. Nguyen, et al., "A distributed energy-harvesting-aware routing algorithm for heterogeneous IoT networks," IEEE Transactions on Green Communications and Networking, vol. 2, no. 4, pp. 1115-1127, 2018.

[23] N. Kumar and D. P. Vidyarthi, "A green routing algorithm for IoT-enabled software defined wireless sensor network," IEEE Sensors Journal, vol. 18, no. 22, pp. 9449-9460, 2018.

[24] W. I. S. W. Din, et al., "An enhancement of path selection to cluster head based on multi-hop routing in two-tier wireless sensor network," International Journal of Electrical \& Computer Engineering (IJECE), vol. 9, no. 6, pp. 5360-5367, 2019.

[25] J. Lin, et al., "Efficient Fault-Tolerant Routing in IoT Wireless Sensor Networks Based on Bipartite-Flow Graph Modeling," IEEE Access, vol. 7, pp. 14022-14034, 2019.

[26] E. Selem, et al., "THE (Temperature Heterogeneity Energy) Aware Routing Protocol for IoT Health Application," IEEE Access, vol. 7, pp. 108957-108968, 2019. 
[27] K. Nagarathna and J. D. Mallapur, "OTSA: Optimized Time Synchronization Approach for Delay-based Energy Efficient Routing in WSN," International Journal of Advanced Computer Science and Applications, vol. 8, no. 4, pp. 331-341, 2017.

[28] K. Nagarathna, et al., "Trust based secured routing in wireless multimedia sensor networks," in 2012 Fourth International Conference on Computational Intelligence, Communication Systems and Networks, pp. 53-58, 2012.

[29] K. Nagarathna and J. D. Mallapur, "An investigational analysis of different approaches and techniques for time synchronization in wireless sensor network," International Journal of Computer Applications, vol. 103, no. 5, pp. 18-28, 2014.

[30] K. Nagarathna and J. D. Mallapur, "TFTS: A Novel Triple Factor Time Synchronization for Effective Routing in Large Scale WSN,” Wireless Personal Communications, vol. 87, no. 4, pp. 1157-1173, 2016.

[31] K. Nagarathna and J. D. Mallapur, "LNLCS: Leveraging Network Lifetime using clock synchronization in wireless sensor network," in 2016 International Conference on Electrical, Electronics, and Optimization Techniques (ICEEOT), pp. 2119-2124, 2016.

[32] A. A. Begam and A. Prema, "Optimization of Eh Aware Routing Protocol for WSN Based Iot Networks," SSRG International Journal of Electronics and Communication Engineering (SSRG - IJECE), pp. 30-35, 2019.

[33] X. Tian, et al., "Reliable and energy-efficient data forwarding in industrial wireless sensor networks," IEEE Systems Journal, vol. 11, no. 3, pp. 1424-1434, 2015.

[34] J. V. V. Sobral, et al., "Improving the Performance of LOADng Routing Protocol in Mobile IoT Scenarios," IEEE Access, vol. 7, pp. 107032-107046, 2019.

[35] B. Manickavasagam, et al., "Optimal packet routing for wireless body area network using software defined network to handle medical emergency," International Journal of Electrical and Computer Engineering (IJECE), vol. 10, no. 1 , pp. 427-437, 2020.

[36] V. D. Chakravarthy and B. Amutha, "Path based load balancing for data center networks using SDN," International Journal of Electrical \& Computer Engineering (IJECE), vol. 9, no. 4, pp. 3279-3285, 2019.

[37] H. T. Zaw and A. H. Maw, "Traffic management with elephant flow detection in software defined networks (SDN)," International Journal of Electrical \& Computer Engineering (IJECE), vol. 9, no. 4, pp. 3203-3211, 2019.

[38] S. N. Hertiana, et al., "Effective Router Assisted Congestion Control for SDN," International Journal of Electrical \& Computer Engineering. (IJECE), vol. 8, no. 6, pp. 4467-4476, 2018.

[39] T. E. Ali, et al., "Load balance in data center SDN networks," International Journal of Electrical and Computer Engineering (IJECE), vol. 8, no. 5, p. 3084-3091, 2018.

\section{BIOGRAPHY OF AUTHOR}



Dr. K. Nagarathna, I am currently working in Basaveshwar Engineering College, Bagalkot, Karnataka, India, as Associate Professor. I have completed my B.E from Vijayanagara Engineering College, Bellary Gulbarga University. I have completed my M.Tech from Poojya Doddappa College of Engineering Gulbarga, from Visvesvaraya Technological University, Belgaum. I have completed Ph.D. (Electronics and Communication Engineering.) Basaveshwar EngineeringCollege, Bagalkot, Karnataka, India, Visvesvaraya Technological University, Belgaum, India. 